\title{
Preparation and Properties of $\operatorname{Mg}\left(\mathrm{B}_{1-\mathrm{X}} \mathrm{C}_{\mathrm{X}}\right)_{2}$ Using Carbon Chemical Vapor Coated Boron
}

\author{
E. A. Young and Y. Yang
}

\begin{abstract}
Promising initial results on bulk $\operatorname{Mg}\left(B_{1-x} C_{x}\right)_{2}$ prepared with carbon doped boron are presented. Carbon doping is achieved by reaction of ethylene gas on boron powder using a stainless steel tube furnace, a technique suitable for industrial scale processing. The nominal amount of doping was controlled by varying the reaction time with a fixed volume of ethylene gas, and the actual carbon uptake was determined by weight change after the reaction. The amount of carbon substitution $x$ in the $\operatorname{Mg}\left(B_{1-x} C_{x}\right)_{2}$ was found using the angular shift in the (100) $x$-ray reflection. Carbon substitution by the full nominal content in the $\mathrm{C}$ doped precursor boron was obtained for doping up to 7.2at\%, as shown by a $a$-axis compression consistent with that of carbon doped single crystals. The critical current density of the 4 at $\% \mathrm{C}$ doped sample for temperatures at 20-30 K and fields up to $4 \mathrm{~T}$, relevant to high temperature applications, was significantly higher than those in the published literature. The $J_{\mathrm{C}}$ of a $10 \mathrm{wt} \%$ nano-SiC doped sample, used as a comparative benchmark, was found to be lower than the $\mathrm{C}$ doped sample at field below $2 \mathrm{~T}$, but to reduce slower at higher fields. Structure analysis of the $\mathrm{SiC}$ doped sample revealed a coexistence of two $\mathrm{C}$ substitution levels of $2.25 \%$ at and $5.25 \%$ at.
\end{abstract}

Index Terms-Boron powder, carbon doping, critical current, $\mathrm{MgB}_{2}$ superconductor.

\section{INTRODUCTION}

$\mathbf{T}$ HE $39 \mathrm{~K}$ transition temperature of $\mathrm{MgB}_{2}$ allows liquidcryogen free technology be used in the $20-30 \mathrm{~K}$ range. Currently NbTi is widely used in many superconducting applications, but requires complex and expensive Liquid He cooling. A realistic $\mathrm{MgB}_{2}$ market will open-up if the critical current in field, $J_{\mathrm{C}}(\mathrm{B})$, performance of $\mathrm{MgB}_{2}$ can be improved to a level where it will compete with $\mathrm{NbTi}$, with a lower cost and smaller size of the cryogenics.

Doping with carbon has so far shown the most promising improvements in the field dependence of the critical current density $J_{\mathrm{C}}(B)$, for both wire and bulk $\mathrm{MgB}_{2}$ conductors, [1], [2]. The improvements in $J_{\mathrm{C}}(B)$ are attributed to lattice defects increasing the upper critical field $H_{\mathrm{C} 2}$. The method of $\mathrm{C}$ distribution in the precursor with uniformity at nanometer scale is therefore essential in order to take full advantage of bulk property enhancements attributed to crystalline defects.

At present carbon has been added successfully by high speed milling, [1] and grinding, [2]. However both methods have the drawback of requiring precursor powder of a small grain size, typically sub-micron nano-particles, which forms

Manuscript received August 29, 2006.

The authors are with the Institute of Cryogenics, School of Engineering Sciences, University of Southampton, Southampton SO17 1BJ, U.K. (e-mail: e.a.young@soton.ac.uk; y.yang@soton.ac.uk).

Digital Object Identifier 10.1109/TASC.2007.897986 soft agglomerates due to the overriding effect of electrostatic attraction at the nanometer length scale. Such agglomerates, difficult to break mechanically, are the source of inhomogeneity detrimental to achieving the uniform doping required. When synthesized to $\mathrm{MgB}_{2}$ the dopants must be transported by diffusion, hence require a higher reaction temperature that may not be desirable for the optimal $J_{\mathrm{C}}(\mathrm{B})$ performance. In addition the long milling times, required for good mixing, of the very hard $\mathrm{MgB}_{2}$ grains with a micro-hardness around $1000 \mathrm{kgmm}^{-2}$ comparable to that of $\mathrm{SiC}$ [3], significantly increase the risk of contamination by the milling media.

In this paper, we describe a method of intrinsically homogeneous carbon loading by chemical vapor deposition (CVD) of carbon on to the boron precursor powder. Such a method presents two advantages. Firstly it allows the dopant to be distributed without contamination. Secondly the dopants are distributed evenly to every individual precursor grains so that a homogeneous nanometer scale distribution can be achieved with a nano-boron powder. The realization of such a CVD carbon loading using ethylene gas in a closed system is described in the paper. Structure analysis was used to determine the level of $\mathrm{C}$ substituted in bulk $\mathrm{MgB}_{2}$ samples prepared with the carbon loaded boron powder by the liquid infiltration technique, [4]. The critical current density and its field dependence $J_{\mathrm{C}}(B)$ were also measured and compared with doping by $\mathrm{SiC}$ nano-particles. The comparison firstly serves to highlight the improvements in critical current relative to another dopant, for the same liquid infiltration technique, and secondly demonstrates that doping by CVD results in more homogenous crystalline properties.

\section{EXPERIMENTAL}

\section{A. Doping of $\mathrm{MgB}_{2}$ Precursor}

A purpose built stainless steel tube furnace was used for the chemical vapor deposition of carbon on boron powder. Ethylene gas was introduced after the furnace tube was evacuated by a turbo-molecular vacuum pump to $10^{-4} \mathrm{mBar}$. Boron powder, with a grain size of $<1 \mu \mathrm{m}$, and a purity of $99.9 \%$ was treated in ethylene gas for fixed periods. The mass of the boron powder was measured before and after the process and the mass of $\mathrm{C}$ deposited estimated from the mass gain. The powders were then examined in a scanning electron microscope (SEM) for morphology analysis. The precursor boron powder CVD coated with carbon is referred as C-CVD boron in the following text, and the corresponding samples as $\mathrm{C}-\mathrm{CVD} \mathrm{MgB}_{2}$.

The nano-SiC doped boron was prepared by mixing the powders together in methanol slurry under ultrasound for several hours, then slowly heated up to $100^{\circ} \mathrm{C}$ for the removal of methanol by evaporation. 


\section{B. Bulk $\mathrm{MgB}_{2}$ Samples}

With the $\mathrm{C}$ doped and $\mathrm{SiC}$ doped precursors $\mathrm{MgB}_{2}$ bulk was made by the liquid infiltration technique, [4]. Boron pellets of $12 \mathrm{~mm}$ diameter and $15 \mathrm{~mm}$ thickness were pressed into Ta lined Fe crucibles. For each sample, a magnesium rod was placed on the top of the pellet. Then the lid of the Fe crucible was closed firmly above the $\mathrm{Mg}$ rod so that no air gap remained, and finally the crucible was sealed by arc welding. Excess $\mathrm{Mg}$ of about $10 \%$ was used to compensate for any loss due to reaction with the Fe and ensure there is sufficient $\mathrm{Mg}$ to complete the formation of magnesium diboride. The Fe crucibles were placed in a vacuum furnace and heated to $850^{\circ} \mathrm{C}$ for $10 \mathrm{~h}$ then furnace cooled to room temperature.

\section{Characterization}

The top face of the reacted pellets were ground back $\sim 3 \mathrm{~mm}$ to present a core surface for $\theta-2 \theta$ powder $\mathrm{x}$-ray diffraction (XRD) analysis. The magnetic hysteresis $\Delta M$ of small slabs, cut with a diamond wheel to a typical dimensions of $\sim 3 \times 1 \times$ $0.25 \mathrm{~mm}^{3}$, were measured in fields up to $9 \mathrm{~T}$ and temperatures between $5 \mathrm{~K}$ and $30 \mathrm{~K}$ using a vibrating sample magnetometer (VSM) in a Quantum Design Physical Property Measurement System. The critical current density was determined using $J_{\mathrm{C}}=$ $20 \Delta M / d$, where $d$ is the thickness of the slab.

\section{RESULTS AND DISCUSSION}

\section{A. Boron Powder CVD Coated With Carbon}

On opening of the crucibles all samples were fully reacted with no $\mathrm{Mg}$ rod remaining, and were removed as hard solid pucks, without large cracks or other macro-scale defects.

Carbon deposition on the boron was confirmed by Energy Dispersive Analysis, which showed no significant changes in the powder morphology shown by Secondary Electron images at a resolution of $\sim 0.1 \mu \mathrm{m}$.

\section{B. Structure Analysis of C-CVD $M g B_{2}$}

Due to a relatively low reaction temperature of $850^{\circ} \mathrm{C}$, the $\mathrm{MgB}_{2}$ pellets obtained were of high phase purity with some $\mathrm{Mg}$ inclusions but no significant secondary $\mathrm{MgB}_{2}$ phases detected by standard XRD analysis. X-ray diffraction of the reacted samples, (Fig. 1), shows increasing C doping shifts the (100) peak, to higher angles, whilst the (002) peak position remains comparatively unchanged. Both peaks broaden considerably with increasing $\mathrm{C}$ content.

The change in the a-axis lattice parameter $\Delta a$, derived from the (100) peak, is plotted in Fig. 2 against the nominal doping fraction $\mathrm{x}$ in the precursor power of $\mathrm{Mg}+2\left(\mathrm{~B}_{1-\mathrm{x}}+\mathrm{C}_{\mathrm{x}}\right)$, calculated from the deposited mass of $\mathrm{C}$, alongside data from the literature [1], [2], [5]. Full carbon substitution at the nominal doping level is confirmed by the agreement of $\Delta a$ between C-CVD $\mathrm{MgB}_{2}$ sample and $\mathrm{C}$ doped single crystal [5]. Therefore the actual doping achieved in $\operatorname{Mg}\left(\mathrm{B}_{1-\mathrm{x}} \mathrm{C}_{\mathrm{x}}\right)_{2}$ equates to the nominal doping fraction $x$ in the precursor powder. The advantage of the present method of $\mathrm{C}$ distribution by CVD coating of $\mathrm{B}$ powder is evident when compared with mechanical carbon doping by grinding [2] and high speed ball milling [1], which only achieved partial substitution of the nominal doping content.

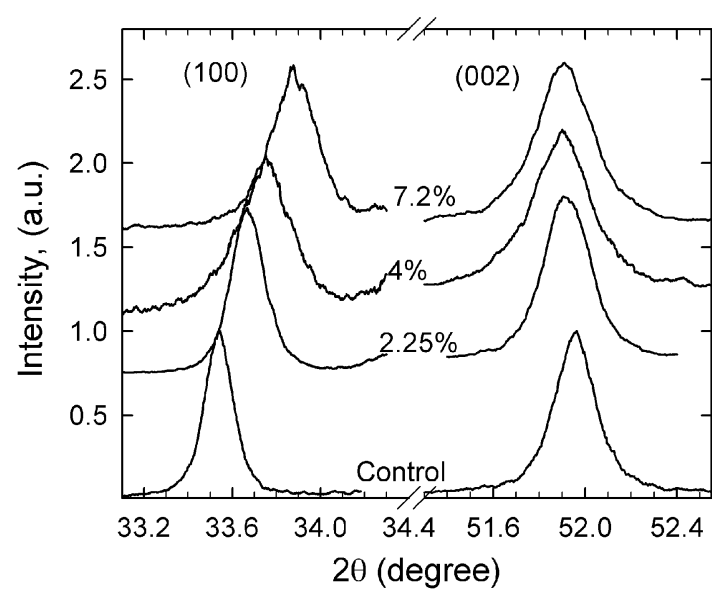

Fig. 1. Reflection of (100) from XRD of the C-CVD doped samples with increasing nominal doping percentage of C. Shift of the reflection to higher angles corresponds to a decreasing a-lattice constant (left), whilst the (002) peak (right) remains comparatively unaffected.

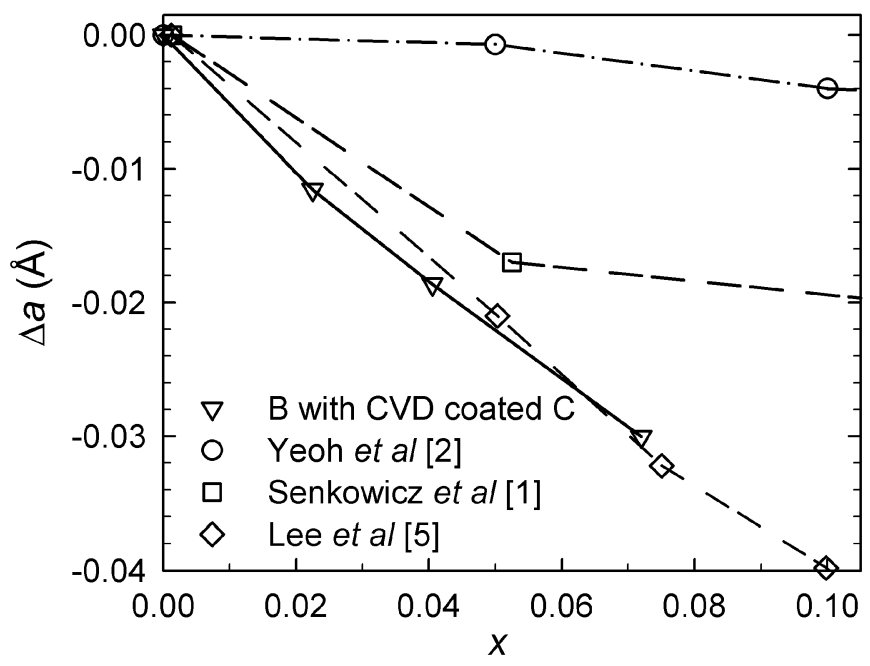

Fig. 2. The change in the a-axis lattice constant, $\Delta$ a calculated from the (100) reflection, against nominal doping fraction $x$, from $\operatorname{Mg}\left(\mathrm{B}_{1-\mathrm{x}} \mathrm{C}_{\mathrm{x}}\right)_{2}$. The compression of $a$-axis $\Delta a$ of the $\mathrm{C}$ doped samples using C-CVD boron are in good agreement with those of $\mathrm{C}$-doped single crystals [5], indicating full substitution of $\mathrm{B}$ by the nominal $\mathrm{C}$ content in the $\mathrm{MgB}_{2}$ lattice. Partial substitution obtained with milling, [1] and grinding [2] are shown for comparison.

As no reduction in $\mathrm{C}$ substitution was found up to $7.2 \%$ at, the technique looks to have the potential for even higher $\mathrm{C}$ doping towards the theoretical saturation limit. The broadening of both (100) and (002) reflections at higher $\mathrm{C}$ doping may be attributed to strain and small grain size. It has been suggested that the lattice disorder is a consequence of the $\mathrm{C}$ prohibiting the crystal growth of the $\mathrm{MgB}_{2}$ [1].

\section{Critical Current Density of C-CVD $\mathrm{MgB}_{2}$}

Fig. 3 shows the critical current density as a function of magnetic field at different temperatures between $5 \mathrm{~K}$ and $30 \mathrm{~K}$. In the $\log -\log$ plot, $J_{\mathrm{C}}(B)$ varies with $\sim 1 / B$ at low fields and then reduces sharply with $\sim 1 / B^{4}$ or exponentially at higher fields. The transition field from one behavior to the other increases with reducing temperature, e.g. $0.75 \mathrm{~T}$ at $30 \mathrm{~K}$ and 4.0 $\mathrm{T}$ at $5 \mathrm{~K}$. Compared with other $\mathrm{C}$ doped $\mathrm{MgB}_{2}$ samples [1], [2], 


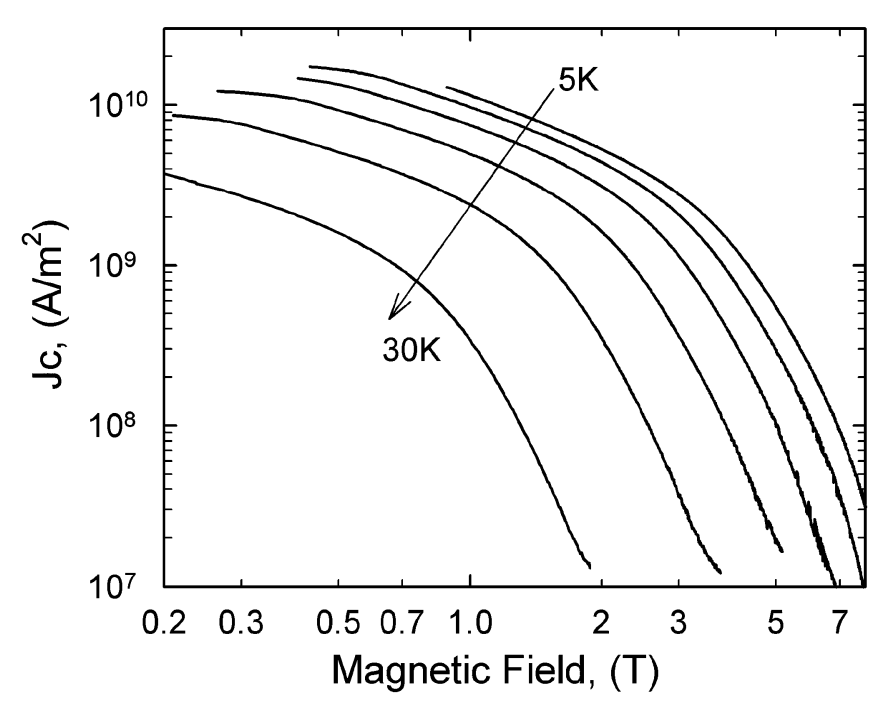

Fig. 3. Critical current density for $4 \%$ at $\mathrm{C}$ doped sample $\left.\mathrm{MgB}_{0.96} \mathrm{C}_{0.04}\right)_{2}$ as a function of magnetic field at temperatures between $5 \mathrm{~K}$ and $30 \mathrm{~K}$ incremented steps of $5 \mathrm{~K}$.

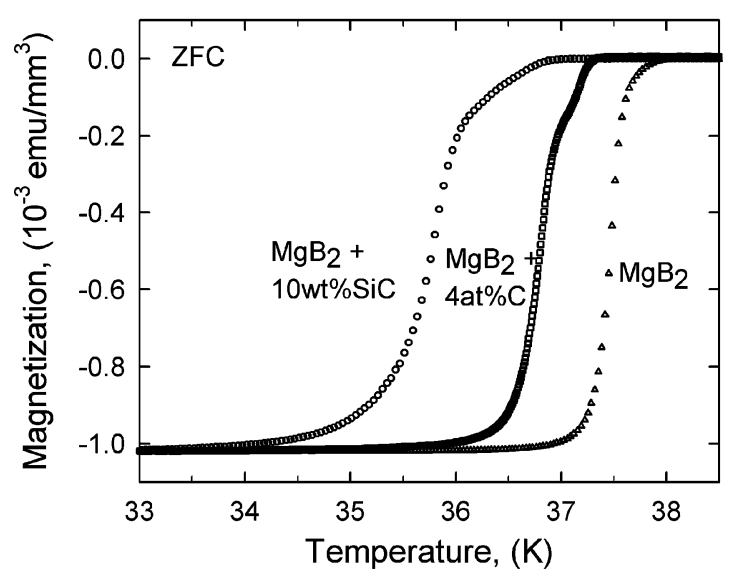

Fig. 4. Zero-field-cooled DC magnetisation of samples made from pure Boron, and Boron doped with $4 \% \mathrm{C}-\mathrm{CVC}$, and 10wt $\%$ nano $\mathrm{SiC}$ doped.

the $J_{\mathrm{C}}$ of the C-CVD $\mathrm{MgB}_{2}$ samples exhibit is most improved at high temperatures and low/intermediate fields. In particular, the $J_{\mathrm{C}}$ at $20-30 \mathrm{~K}$ and in fields up to $4 \mathrm{~T}$ is among the highest of any $\mathrm{MgB}_{2}$ preparation in the published literature. A better performance principally at high temperatures and lower field indicates a clean $\mathrm{C}$ substitution without significant formation of secondary phases, as also shown by the XRD data in Fig. 1. It is also noted that the critical temperature $T_{\mathrm{C}}$ of the $4 \%$ at C-CVD sample was at $36.6 \mathrm{~K}$ (Fig. 4), which is significantly higher those at 30-33 $\mathrm{K}$ for $\mathrm{C}$ doped single crystals samples [4], [5] with the same a-lattice reduction of 0.02 Á. The apparent non-commensurate nature of $T_{\mathrm{C}}$ with a-axis lattice parameter may be symptomatic of a separation of structural shift and electronic doping, and will be a topic of further investigation. At this early stage in this work it is worth pointing out that the single crystals were grown at $1600-1900^{\circ} \mathrm{C}$, whilst the C-CVD samples in this work were reacted at $850^{\circ} \mathrm{C}$. It is possible that the reaction temperature affects nature of the $\mathrm{C}-\mathrm{B}$ and $\mathrm{C}-\mathrm{Mg}$ bonds.

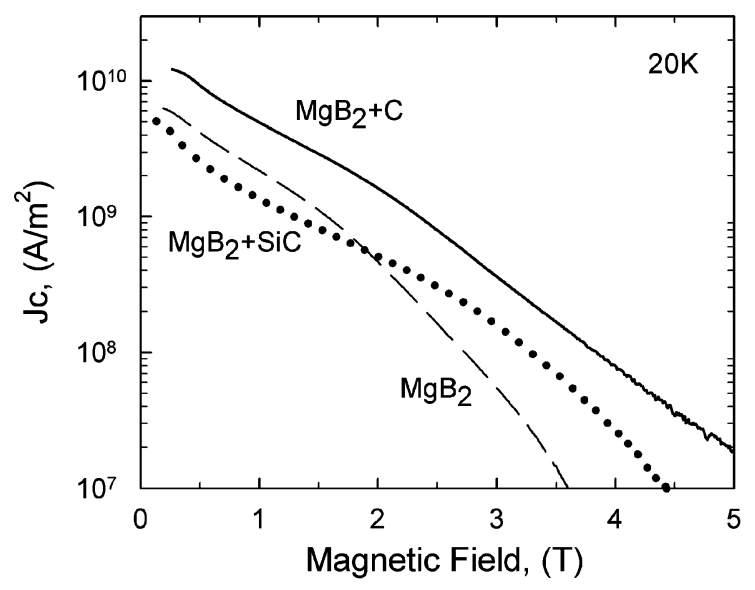

Fig. 5. Comparison of critical current at $20 \mathrm{~K}$ for samples made from pure boron, and boron doped with $4 \% \mathrm{C}-\mathrm{CVD}$, and $10 \mathrm{wt} \%$ nano $\mathrm{SiC}$ doped.

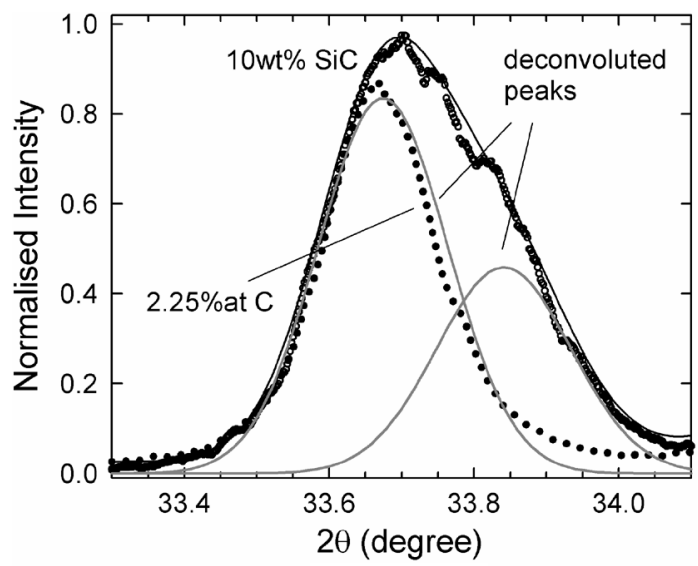

Fig. 6. The (100) reflection of the $10 \mathrm{wt} \%$ nano-SiC, (symbols), deconvoluted, reveals it is composed of two reflections, (dashed lines), the low angle is near identical to the measured 2.25\% C-CVD sample, (solid line), and the higher angle peak can be interpreted from a linear interpolation of C-CVD doped data in Fig. 2 as $5.25 \% \mathrm{C}$ inclusion.

\section{Comparison Between Carbon Doping by C-CVD Boron and Nano-SiC}

In Fig. 5 the $J_{\mathrm{C}}(B)$ at $20 \mathrm{~K}$ of the $4 \% \mathrm{C}-\mathrm{CVD}$ doped $\mathrm{MgB}_{2}$ sample is compared with that of $10 \mathrm{wt} \% \mathrm{SiC}$ doped sample, with the un-doped control sample shown as the reference. The C-CVD doped sample has a better performance throughout the field range. It is noted, however, that the $\mathrm{SiC}$ doped sample showed a slower $J_{\mathrm{C}}$ reduction between $0.5 \mathrm{~T}$ and $3 \mathrm{~T}$, possibly indicating contribution of a different pinning mechanism in this field range.

It has been suggested [1] that improvements in $J_{\mathrm{C}}(B)$ by $\mathrm{SiC}$ doping are due to $\mathrm{C}$ doping of the $\mathrm{MgB}_{2}$. While the (100) reflection of the $\mathrm{SiC}$ doped sample shown in Fig. 6 is shifted to a high angle as expected, the broad non-symmetric peak is significantly different from those found in the C-CVD samples. Two underlying Gaussian peaks were found by $\chi^{2}$ fitting (solid line). The peak at lower angle is very close to that of $2.3 \%$ at C-CVD doping (solid dots), while the higher angle peak corresponds to a $\mathrm{C}$ doping percentage of about $5 \%$ at using the C-CVD and single crystal data shown Fig. 2. The split of the (100) peak into two peaks can be interpreted as an inhomogenous lattice structure 
with non-uniform $\mathrm{C}$ doping by $\mathrm{SiC}$. Different $\mathrm{C}$ substitution level in $\mathrm{SiC}$ doped samples is most likely the result of the inhomogeneity in nano-SiC distribution in the precursor powder. In contrast, the relatively clean XRD found in C-CVD samples makes it ideal to identify the contribution directly resulted from lattice substation by $\mathrm{C}$, reducing the complications of a dirtier system such as SiC doping. A broad two step superconducting transition in SiC doped sample (Fig. 4) with an onset $T \mathrm{C}$ as high as $36.4 \mathrm{~K}$ also underlines multiple $\mathrm{C}$ doping levels suggested by the XRD data.

Further work to measure the $J_{\mathrm{C}}(B)$ of samples made with a range of C-doping percentages will allow the possible interpretation of the $\mathrm{SiC}$ doped sample into two parallel current paths of different $\mathrm{C}$ doping levels.

\section{CONCLUSION}

Initial results on the physical and magnetic properties of $\mathrm{MgB}_{2}$ bulk made from C-CVD doped B. Full inclusion of $\mathrm{C}$ has been demonstrated for samples of $\mathrm{Mg}\left(\mathrm{B}_{1-\mathrm{x}} \mathrm{C}_{\mathrm{x}}\right)_{2} \mathrm{x}=0.02$, 0.04 , and 0.07. At this stage the optimum percentage of $\mathrm{C}$ for maximum $J_{\mathrm{C}}(B)$ has not yet been found, but already the
4\% sample has the highest critical currents available in the literature in the 20-30 K, 1-4 $\mathrm{T}$ range, where the first $\mathrm{MgB}_{2}$ applications are currently anticipated. By comparing the XRD and $J_{\mathrm{C}}$ properties of the $\mathrm{C}-\mathrm{CVD}$ doped to a nano-SiC doped sample, the dispersion of $\mathrm{C}$ is shown to be better by the CVD route and the critical current consistently higher.

\section{REFERENCES}

[1] B. J. Senkowicz, J. E. Giencke, S. Patnaik, C. B. Eom, E. E. Hellstrom, and D. C. Larbalestier, "Improved upper critical field in bulk-form magnesium diboride by mechanical alloying with carbon," Appl. Phys Letts, vol. 86, pp. 202502-202504, 2005.

[2] W. K. Yeoh, J. H. Kim, J. Horvat, X. Xu, M. J. Qin, S. X. Dou, C. H. Jiang, T. Nakane, H. Kumakura, and P. Munroe, "Control of nano carbon substitution for enhancing the critical current density in MgB2," Supercond. Sci. Technol., vol. 19, pp. 596-599, 2006.

[3] S. M. Kazakov, J. Karpinski, J. Jun, P. Geiser, N. D. Zhigadlo, R. Puzniak, and A. V. Mironov, "Single crystal growth and properties of $\mathrm{MgB} 2$ and $\mathrm{Mg}\left(\mathrm{B} 1 \_\mathrm{xCx}\right) 2$," Physica C, vol. 408-410, pp. 123-124, 2004.

[4] G. Giunchi, G. Ripamonti, T. Cavallin, and E. Bassani, "The reactive liquid $\mathrm{Mg}$ infiltration process to produce large superconducting bulk MgB2 manufacts," Cryogenics, vol. 46, pp. 237-242, 2006.

[5] S. Lee, T. Masui, A. Yamamoto, H. Uchiyama, and S. Tajima, "Carbonsubstituted MgB2 single crystals," Physica C, vol. 397, pp. 7-13, 2003. 